\title{
One-loop renormalization of Lorentz and $C P T$-violating scalar field theory in curved spacetime
}

\author{
Tibério de Paula Netto* \\ Departamento de Física, ICE, Universidade Federal de Juiz de Fora, 36036-330 MG, Brazil
}

(Received 13 December 2017; published 29 March 2018)

\begin{abstract}
The one-loop divergences for the scalar field theory with Lorentz and/or CPT breaking terms are obtained in curved spacetime. We analyze two separate cases: a minimal coupled scalar field with gravity and a nonminimal one. For the minimal case with a real scalar field, the counterterms are evaluated in a nonperturbative form in the $C P T$-even parameter through a redefinition of a space-time metric. In the most complicated case of a complex scalar field nonminimally interacting with gravity, the solution for the divergences is obtained in the first order in the weak Lorentz violating parameter. The necessary form of the vacuum counterterms indicate the most important structures of Lorentz and $C P T$ violations in the pure gravitational sector of the theory. The conformal theory limit is also analyzed. It turns out that if we allow the violating fields to transform, the classical conformal invariance of massless scalar fields can be maintained in the $\xi=1 / 6$ case. At a quantum level, the conformal symmetry is violated by a trace anomaly. As a result, the conformal anomaly and the anomaly induced effective action are evaluated in the presence of extra Lorentz- and/or CPT-violating parameters. Such gravitational effective action is important for cosmological applications and can be used for searching of Lorentz violation in the primordial Universe in the cosmological perturbations, especially gravitational waves.
\end{abstract}

DOI: $10.1103 /$ PhysRevD.97.055048

\section{INTRODUCTION}

In the last years, there was an intensive investigation of the theoretical and experimental aspects in theories where the Lorentz and/or $C P T$ symmetries are violated. Such violations could emerge in a number of different fashions, most of them related to a new physics at the Planck scale $M_{P} \simeq 10^{19} \mathrm{GeV}$. As examples, we can cite quantum effects in string theory [1] and loop quantum gravity [2], geometrical effects as noncommutativity [3], torsion [4], and nonmetricity [5]; and so on [6]. Regardless of wherever these violations might come from, or if exposed scenarios may or may not occur, the experimental/observational searching of the remaining small deviations from Lorentz and $C P T$ symmetries in nowadays attainable scales are of crucial importance, representing at the moment a very active area of physics [7]. Such considerations can indicate the existence of new physical phenomena or, at least, improve our understanding of the limits of validity of the current one.

The conventional approach for this type of consideration starts, of course, with theoretical aspects, considering the

\footnotetext{
*tiberiop@fisica.ufjf.br
}

Published by the American Physical Society under the terms of the Creative Commons Attribution 4.0 International license. Further distribution of this work must maintain attribution to the author(s) and the published article's title, journal citation, and DOI. Funded by SCOAP ${ }^{3}$. most general consistent form of Lorentz and $C P T$ violating terms in the action of quantum matter fields. Such a theory is called the standard model extension (SME) [8]. The action of the SME contains all the possible new operators that parametrize the possible Lorentz and/or $C P T$ violations which can be constructed from scalars, fermions, and vectors fields using the effective quantum field theory (QFT) approach. After that, as a next step, one can look for the possible phenomenological manifestation of these new terms. For the updated date table of bounds on Lorentz/CPT breaking terms and some experimental details, see [9] and further references therein. Likewise, from the QFT perspective, the presence of additional background fields means that the modifications may occur not only at a classical level but also at the quantum one. The investigation of flat spacetime loop effects in Lorentz and $C P T$ violated quantum electrodynamics (QED) started in the pioneer work [10], where the corresponding quantum effects were derived and new bounds on the violating parameter were indicated.

From the pure gravitational side, there is also the possibility for Lorentz violation in the SME [11]. Nonetheless, in the vacuum sector of Lorentz and CPT violating theories, one can introduce terms with these symmetry breaking in many distinct ways. Besides the linear in curvature violating terms, there is additionally the possibility to introduce a huge amount of higher derivative structures in the gravitation action. Indeed, the pure 
gravitational sector with the Lorentz violation can be described by an action containing an infinite series of higher derivative terms with operators of increasing mass dimension. As a concrete example, we can cite the theories with torsion. In this case, the general vacuum action of gravity, which contains only a small part of CPT and Lorentz violating terms, includes an incredible 168 independent terms [12]. Such great arbitrariness makes it very difficult to choose which of those terms are the most important ones, and therefore, a natural question is how to define the form of the possible Lorentz and/or $C P T$ symmetries violation in the gravitational sector.

One of the possibilities is to introduce only those terms which can emerge as vacuum divergences in a semiclassical theory of quantum matter fields. These criteria concern the minimal necessary set of terms in the classical gravitational action which are required by renormalizability. One example where this approach is widely applied is the QFT in curved spacetime. In this case, the renormalizability is achieved by introducing along with the Einstein-Hilbert and cosmological constant terms, a full set of local covariant fourth derivative structures (for an introduction on this subject see, e.g. the textbook [13]). For the theories with Lorentz- and/or $C P T$-violating terms, one meets an analogous situation. The introduction of new terms in the matter fields sector requires, at the quantum level, the extension of a classical action of vacuum with a set of structures depending on the violating parameters. The form of those terms may be established on the basis of general covariance and power counting arguments, but only direct calculation of the counterterms can indicate which terms are truly necessary. And since the Lorentz and $C P T$ breaking terms are very small, the one-loop calculation are the most important ones. Hence, our strategy to investigate the possible role of the violating parameters in the gravitational sector is to start by deriving the one-loop divergences for the SME fields on a curved background.

The first consideration in this direction was presented in Ref. [14] (see also [15]). In this work, the one-loop calculations in the Lorentz and $C P T$ violating QED were considered in curved spacetime. However, the presented calculations were not complete, because only the divergences of effective action (EA), which concern to the minimal part of the corresponding bilinear operator, have been taken into account. After that, the problem of working with the complicated nonminimal structures, which appears typically in the EA of Lorentz and $C P T$ violating theories, was solved in Ref. [16] by introducing a new calculation trick involving the inversion of the minimal part of a bilinear operator. Working at first order in the violating parameters, the complete photon contribution to vacuum renormalization was obtained. In this work, the corresponding EA of gravity was also derived by the integration of conformal anomaly. This anomaly induced EA corresponds to the leading quantum contribution for present-day low energy physics applications, since the photon is the lightest field, and the other massive fields suffer from the Appelquist and Carazzone decoupling theorem [17], which takes place, also, for the vacuum gravitational sector [18]. However, for the interesting cosmological applications in the early Universe, in which the typical energies of physical phenomena are very large, all other quantum matter fields provides quantum contributions which are as important as the photon, since in this high energy situation, matter behaves approximately as free radiation. Consequently, the evaluation of loop effects in a curved background coming from the other SME sectors is also a relevant question.

In the present work, we report the results of the one-loop counterterms calculations for the Lorentz and $C P T$ violating massive complex scalar field theory in curved spacetime. The effects in the vacuum renormalization of the adimensional $C P T$-even violating field and mass dimension $C P T$ odd parameter are analyzed. Furthermore, we also consider the possibility of nonminimal interaction of scalars with gravity in the form $\xi R \varphi^{*} \varphi$. The inclusion of a nonminimal term is necessary for the renormalizability of an interacting theory which includes scalars without Lorentz and/or $C P T$ violating terms (see, e.g. [13,19] for the introduction). Furthermore, the nonminimal parameter $\xi$ plays an important role to inflationary models such as Higgs inflation, where the nonminimal $\xi R H^{\dagger} H$ term is added to the Higgs potential $[20,21]$. It seems natural to extend these theories to the quantum level, studying the possible interaction between the nonminimal parameter and the Lorentz/CPT violating fields, especially because we know that quantum effects are essential in the scalar inflaton models.

The introduction of a nonminimal interaction also opens the way to the study the massless conformal theory limit and conformal anomaly $[22,23]$. The integration of the anomaly yields the anomaly induced effective action of gravity [24], which is a compact analytic form of quantum corrections. The anomaly induced EA has many useful applications in cosmological models such as the full Starobinsky model of inflation [25] or its modified version [26]. The primordial Universe could be seen as a subject of very special interest for the Lorentz and $C P T$ symmetries violating theories, because it can be considered as a laboratory for the study of physical phenomena in energy scales not currently available in particle accelerators on Earth. Moreover, the early Universe may have been very different from its presentday situation, because since then some kind of physical process of spacetime symmetry restoration may have occurred. We expected that the Lorentz breaking terms make no effect on the zero-order homogeneous and isotropic cosmology, since the violating fields define a preferable direction in the spacetime. However, many of the symmetrybreaking terms may lead to anisotropy in the cosmic microwave radiation [27] coming from the cosmic perturbations in the inflationary epoch. Therefore, it would be interesting to evaluate the possibility of such violations, in 
particular, in the primordial Universe with gravitational waves calculations.

The organization of the paper is as follows. In Sec. II, the one-loop divergences for Lorentz- and/or $C P T$-violating scalar field theory are derived in curved spacetime. We consider separately minimal and nonminimal interaction with gravity. In both cases, we adopt dimensional regularization, and the curved background calculations are performed by means of the heat kernel techniques related with the Schwinger-DeWitt method [28,29]. Hence, the minimal set of pure gravitational terms requested by renormalizability is also analyzed. In Sec. III, the local conformal symmetry limit is investigated in the presence of the symmetry-breaking terms, and from the results obtained in the previous section, the conformal anomaly is calculated. Section IV is devoted to integrating conformal anomaly and, therefore, the gravitational anomaly induced effective action is derived. On the technical side, most of the considerations in this section are pretty well known, and the standard procedure do not change so much in the presence of Lorentz violating fields, but we present adequate details in order to make it readable for those not trained within this subject. Finally, in Sec. V, we draw our conclusions.

Our sign conventions are $\eta_{\mu \nu}=\operatorname{diag}(+,-,-,-)$ for the Minkowski spacetime metric and $R_{. \beta \mu \nu}^{\alpha}=\partial_{\mu} \Gamma_{\beta \nu}^{\alpha}-\cdots$ for the Riemann tensor. The Ricci tensor is $R_{\mu \nu}=R_{. \mu \alpha \nu}^{\alpha}$, and $R=g^{\mu \nu} R_{\mu \nu}$ denotes the Ricci scalar curvature. We also assume that the spacetime is torsionless and use spatial distance and mass definitions such that $c=\hbar=1$.

\section{DERIVATION OF ONE-LOOP DIVERGENCES}

Our model of interest is the massive complex scalar field theory with extra Lorentz and $C P T$ symmetry-breaking terms. The extension for the curved background is obtained by the nonminimal procedure of covariant generalization. The corresponding action has the form

$$
\begin{aligned}
S= & \int d^{4} x \sqrt{-g}\left\{g^{\mu \nu} \partial_{\mu} \varphi^{*} \partial_{\nu} \varphi-m^{2} \varphi^{*} \varphi+\xi R \varphi^{*} \varphi\right. \\
& \left.+K^{\mu \nu}(x) \partial_{\mu} \varphi^{*} \partial_{\nu} \varphi+k^{\mu}(x) j_{\mu}\right\},
\end{aligned}
$$

where

$$
j_{\mu}=i\left(\varphi^{*} \partial_{\mu} \varphi-\varphi \partial_{\mu} \varphi^{*}\right)
$$

is the scalar field current. The $\xi R \varphi^{*} \varphi$ term is called nonminimal, and the parameter $\xi$ is known as the nonminimal parameter. The dimensionless $K^{\mu \nu}(x)$ term is the Lorentz $C P T$-even violating second-rank tensor, and $k^{\mu}(x)$ is the Lorentz $C P T$-odd breaking parameter with mass dimension. Since we are working in a curved background, we do not consider these parameters constants and, hence, we will let them being local functions of the spacetime coordinates. The $x$ dependence also removes the known arbitrariness in the $C P T$-odd $k^{\mu}$ term. ${ }^{1}[11,30]$. No one assumption about the violating parameters is made. ${ }^{2}$ The role of Lorentz and CPT symmetry breaking terms in the scalar sector of SME was widely studied in the flat spacetime limit. The first bounds on the symmetrybreaking terms for the Higgs field were obtained in [31], and quantum loops effects were considered in Refs. [32-36]. For further effects in violating scalar field theories, see also the applications in the Yukawa potential [30], effective potential [37], Casimir effect [38], defect structures [39], and Bose-Einstein condensates [40].

In what follows, we consider the calculation of one-loop divergences in two separate cases. First, we consider the minimal $\xi=0$ theory with a real scalar field. The reason is because in this case, the current $i\left(\varphi^{*} \partial_{\mu} \varphi-\varphi \partial_{\mu} \varphi^{*}\right)$ drops out, and the tensor $K^{\mu \nu}$ is necessarily a symmetric tensor. Therefore, for this simpler situation, it is possible through a redefinition of the metric tensor to obtain a closed answer for the counterterms which is valid to all orders in the Lorentz violation parameter. After that, we are going to consider the general case described by the full theory (1). For this more complicated case, we are going to restrict our calculations to the first order in the symmetry-breaking parameters.

\section{A. One-loop divergences: Minimal coupling with gravity}

As a first example, consider the massive real scalar field minimally coupled with gravity. In this case, the whole expression for the action reads

$$
S=\frac{1}{2} \int d^{4} x \sqrt{-g}\left\{\left(g^{\mu \nu}+K^{\mu \nu}\right) \partial_{\mu} \varphi \partial_{\nu} \varphi-m^{2} \varphi^{2}\right\}
$$

In order to evaluate the one-loop divergences, let us define a new metric,

$$
G^{\mu \nu}=g^{\mu \nu}+K^{\mu \nu} .
$$

After that, the action (3) becomes

$$
S=\frac{1}{2} \int d^{4} x \sqrt{-G} f(x)\left(G^{\mu \nu} D_{\mu} \varphi D_{\nu} \varphi-m^{2} \varphi^{2}\right),
$$

where $G=\operatorname{det}\left(G_{\mu \nu}\right)$ is the determinant of the metric $G_{\mu \nu}$, defined as the inverse to $G^{\mu \nu}$, and

\footnotetext{
${ }^{1}$ For constant $k^{\mu}$ in Minkowski spacetime, the field reparametrization

$$
\varphi(x) \rightarrow \varphi(x) \cdot e^{i \bar{k}^{\mu} x_{\mu}}, \quad \varphi^{*}(x) \rightarrow e^{-i \bar{k}^{\mu} x_{\mu}} \cdot \varphi^{*}(x),
$$

with $\bar{k}^{\mu}=\left(\eta^{\mu \nu}+K^{\mu \nu}\right)^{-1} k_{\nu}$ leads to a new theory without the $C P T$ odd $k^{\mu} j_{\mu}$ term, but with a new mass definition $m^{2} \rightarrow m^{2}+\bar{k}_{\mu} \bar{k}^{\mu}$.

${ }^{2}$ Of course, for the Lagrangian (1) to be real and a scalar quantity, the parameter $K^{\mu \nu}$ must possess, in general, a real symmetric part plus an antisymmetric imaginary one.
} 


$$
f(x)=\sqrt{\frac{\operatorname{det}\left(g^{\mu \nu}+K^{\mu \nu}\right)}{\operatorname{det}\left(g^{\mu \nu}\right)}}
$$

is a new background scalar field. Also, here $D_{\mu}$ is the covariant derivative constructed with the affine connection

$$
\Upsilon_{\alpha \beta}^{\tau}=\frac{1}{2} G^{\tau \lambda}\left(\partial_{\alpha} G_{\lambda \beta}+\partial_{\beta} G_{\alpha \lambda}-\partial_{\lambda} G_{\alpha \beta}\right)
$$

written in terms of the new metric. In the following related calculations, the indexes are lowered and raised with $G_{\mu \nu}$ and with its inverse. It is also very useful to introduce the corresponding curvature tensor

$$
\left[D_{\mu}, D_{\nu}\right] A^{\alpha}=\mathcal{K}_{. \beta \mu \nu}^{\alpha} A^{\beta},
$$

and its contractions $\mathcal{K}_{\alpha \beta}=G^{\mu \nu} \mathcal{K}_{\mu \alpha \nu \beta}$ and $\mathcal{K}=G^{\mu \nu} \mathcal{K}_{\mu \nu}$. These new curvatures differ from the usual Riemann, Ricci tensors, and scalar curvature $R$ by terms of first and higher orders in the Lorentz violating parameter $K^{\mu \nu}(x)$.

The procedure described above is a known calculation method which is commonly used in the Lorentz violating real scalar field theory [30,32]. However, since here the Lorentz violating parameter $K^{\mu \nu}$ is not constant, the answer will not be given only in terms of $K^{\mu \nu}$ determinants, as it was in the flat spacetimes cases, but also in terms of the new curvature tensor $\mathcal{K}_{. \beta \mu \nu}^{\alpha}$. The same method was also recently applied in Ref. [41] for the quantization of the Stueckelberg scalar sector of the massive vector field theory with nonminimal coupling with gravity.

Starting from Eq. (5), the divergences derivation becomes pretty much standard. The divergent part of the one-loop effective action is given by the expression

$$
\Gamma_{\mathrm{div}}^{(1)}=\left.\frac{i}{2} \operatorname{Tr} \ln \left(D^{2}+2 \hat{l}^{\mu} D_{\mu}-m^{2}\right)\right|_{\mathrm{div}},
$$

where

$$
\hat{l}_{\mu}=\frac{1}{2} \partial_{\mu}(\ln f) \quad \text { and } \quad D^{2}=G^{\mu \nu} D_{\mu} D_{\nu} .
$$

The expression in Eq. (9) can be evaluated by means of the standard Schwinger-DeWitt technique [28]. According to this method, the algorithm for the calculation of one-loop divergences, in dimensional regularization, is

$$
\begin{aligned}
\Gamma_{\mathrm{div}}^{(1)}= & -\frac{\mu^{n-4}}{\epsilon} \int d^{n} x \sqrt{-G}\left[\frac{1}{180}\left(\mathcal{K}_{\mu \nu \alpha \beta}^{2}-\mathcal{K}_{\alpha \beta}^{2}+D^{2} \mathcal{K}\right)\right. \\
& \left.+\frac{1}{2} \hat{P}_{\min }^{2}+\frac{1}{6} D^{2} \hat{P}_{\min }\right]
\end{aligned}
$$

where $\epsilon=(4 \pi)^{2}(n-4)$ is the dimensional regularization parameter, $\mu$ is the mass dimensional parameter of renormalization, and

$$
\hat{P}_{\min }=-m^{2}+\frac{1}{6} \mathcal{K}-D_{\mu} \hat{l}^{\mu}-\hat{l}_{\mu} \hat{l}^{\mu}
$$

From Eq. (12), we obtain

$\frac{1}{2} \hat{P}_{\text {min }}^{2}=\frac{1}{2} m^{4}-\frac{1}{6} m^{2} \mathcal{K}+\frac{1}{72} \mathcal{K}^{2}-m^{2} F+\frac{1}{6} \mathcal{K} F+\frac{1}{2} F^{2}$,

where in the last expression, we introduced the useful new notation

$$
F=-\frac{1}{\sqrt{f}}\left(D^{2} \sqrt{f}\right)
$$

Thus, the one-loop divergences can be written in the form

$$
\begin{aligned}
\Gamma_{\text {div }}^{(1)}= & -\frac{\mu^{n-4}}{\epsilon} \int d^{n} x \sqrt{-G}\left\{\frac{1}{180} \mathcal{K}_{\alpha \beta \mu \nu}^{2}-\frac{1}{180} \mathcal{K}_{\alpha \beta}^{2}\right. \\
& +\frac{1}{30} D^{2} \mathcal{K}+\frac{1}{72} \mathcal{K}^{2}-\frac{1}{6} m^{2} \mathcal{K}+\frac{m^{4}}{2}+\frac{1}{6} \mathcal{K} F \\
& \left.-m^{2} F+\frac{1}{2} F^{2}+\frac{1}{6} D^{2} F\right\} .
\end{aligned}
$$

The expression (15) is the result of a standard QFT calculation in the theory with the new background metric $g^{\mu \nu}+K^{\mu \nu}$. In terms of this new metric, formula (15) has a rather standard form. At the same time, in terms of the original fields, $g_{\mu \nu}$ and $K_{\mu \nu}$, the divergences are given by an infinite series expression. This is an expected result which is corroborated by power counting based arguments, since the Lorentz violating parameter $K^{\mu \nu}$ is dimensionless.

The Eq. (15) enables us to obtain the one-loop divergences in terms of the original metric $g_{\mu \nu}$ in each desired order in the Lorentz violating parameter $K^{\mu \nu}(x)$. To obtain the explicit expression for the leading first order, we can use the standard expansions

$$
\begin{aligned}
G_{\mu \nu}= & g_{\mu \nu}-K_{\mu \nu}+\cdots, \\
\sqrt{-G}= & \sqrt{-g}\left(1-\frac{1}{2} K+\cdots\right), \\
\mathcal{K}_{\mu \nu \alpha \beta}= & R_{\mu \nu \alpha \beta}+\frac{1}{2}\left(\nabla_{\mu} \nabla_{\alpha} K_{\beta \nu}-\nabla_{\nu} \nabla_{\alpha} K_{\beta \mu}\right. \\
& +\nabla_{\nu} \nabla_{\beta} K_{\alpha \mu}-\nabla_{\mu} \nabla_{\beta} K_{\alpha \nu}+R_{. \alpha \mu \nu}^{\rho} K_{\rho \beta} \\
& \left.-R_{. \beta \mu \nu}^{\rho} K_{\rho \alpha}\right)+\cdots,
\end{aligned}
$$

where $K=g^{\mu \nu} K_{\mu \nu}$. For a more detailed exposition of the first-order formulas, see [41]. Using those expansions, we can find the one-loop divergences written down in terms of the original metric $g_{\mu \nu}$ in the first order in the Lorentz violating parameter 


$$
\begin{aligned}
\Gamma_{\mathrm{div}}^{(1)}= & \Gamma_{\mathrm{vac}}^{(1)}\left[g_{\mu \nu}\right]-\frac{\mu^{n-4}}{\epsilon} \int d^{n} x \sqrt{-g}\left\{\frac{1}{60} R_{\mu \nu} \square K^{\mu \nu}-\frac{1}{30} R \nabla_{\mu} \nabla_{\nu} K^{\mu \nu}+\frac{1}{90} K^{\mu \nu} R^{\alpha \beta} R_{\alpha \mu \beta \nu}\right. \\
& +\frac{1}{90} K^{\mu \nu} R_{\mu \rho \alpha \beta} R_{\nu}^{\rho \alpha \beta}-\frac{1}{45} K^{\mu \nu} R_{\mu \alpha} R_{\nu}^{\alpha}+\frac{1}{36} K^{\mu \nu} R R_{\mu \nu}+\frac{m^{2}}{6} K^{\mu \nu} R_{\mu \nu} \\
& \left.-\frac{K}{2}\left[\frac{1}{180} R_{\mu \nu \alpha \beta}^{2}-\frac{1}{180} R_{\mu \nu}^{2}+\frac{1}{30} \square R+\frac{1}{72} R^{2}+\frac{m^{2}}{6} R+\frac{m^{4}}{2}\right]\right\},
\end{aligned}
$$

where

$$
\Gamma_{\mathrm{vac}}^{(1)}\left[g_{\mu \nu}\right]=-\frac{\mu^{n-4}}{\epsilon} \int d^{n} x \sqrt{-g}\left\{\frac{1}{180} R_{\mu \nu \alpha \beta}^{2}-\frac{1}{180} R_{\mu \nu}^{2}+\frac{1}{30} \square R+\frac{1}{72} R^{2}+\frac{m^{2}}{6} R+\frac{m^{4}}{2}\right\}
$$

is the divergent part of the pure metric dependent vacuum effective action of a minimally coupled scalar field (see, e.g. $[13,19]$ ). For the sake of brevity, in Eq. (17) (and in the following formulas), we disregarded the total derivative terms in the Lorentz- and/or CPT-violating sector.

\section{B. One-loop divergences: Nonminimal coupling with gravity}

Let us consider now the case of a nonminimally coupled with gravity Lorentz and $C P T$ violating complex massive scalar field with the general nonminimal coupling parameter $\xi$. The action (1) can be cast in the bilinear form

$$
S=-\frac{1}{2} \int d^{4} x \sqrt{-g}\left(\begin{array}{ll}
\varphi & \left.\varphi^{*}\right) \hat{\mathbf{H}} \\
\varphi
\end{array}\right)
$$

where due to the presence of the extra adimensional Lorentz violating parameter $K^{\mu \nu}(x)$, the differential bilinear operator $\hat{\mathbf{H}}$ has a nonstandard general nonminimal structure, namely,

$$
\hat{\mathbf{H}}=\hat{\mathbf{H}}_{m}+\hat{\mathbf{H}}_{n m},
$$

where

$$
\hat{\mathbf{H}}_{m}=\hat{\mathbf{1}} \square+2 \hat{\mathbf{L}}^{\mu} \nabla_{\mu}+\hat{\mathbf{\Pi}}
$$

is the minimal part of bilinear operator in quantum fields and

$$
\hat{\mathbf{H}}_{n m}=\hat{\mathbf{K}}^{\mu \nu} \nabla_{\mu} \nabla_{\nu}
$$

is the nonminimal part. The relevant matrices are defined by

$$
\begin{aligned}
\hat{\mathbf{1}} & =\left(\begin{array}{ll}
1 & 0 \\
0 & 1
\end{array}\right), \\
\hat{\mathbf{L}}^{\mu} & =\left(\begin{array}{cc}
\frac{1}{2} \nabla_{\nu} K^{\mu \nu}+i k^{\mu} & 0 \\
0 & \frac{1}{2} \nabla_{\nu} K^{\mu \nu}-i k^{\mu}
\end{array}\right), \\
\hat{\mathbf{\Pi}} & =\left(\begin{array}{cc}
m^{2}-\xi R & 0 \\
0 & m^{2}-\xi R
\end{array}\right), \\
\hat{\mathbf{K}}^{\mu \nu} & =\left(\begin{array}{cc}
K^{\mu \nu} & 0 \\
0 & K^{\mu \nu}
\end{array}\right) .
\end{aligned}
$$

Here and in the following, we use bold notations for the matrix operators only.

Thus, the one-loop divergent part of the effective action is then given by

$$
\Gamma_{\mathrm{div}}^{(1)}=\left.\frac{i}{2} \ln \operatorname{Det} \hat{\mathbf{H}}\right|_{\mathrm{div}}=\left.\frac{i}{2} \operatorname{Tr} \ln \hat{\mathbf{H}}\right|_{\mathrm{div}} .
$$

Our next purpose is derive the divergent expression (24) through heat kernel related calculations. However, the bilinear operator (20) has a nonminimal form because of the presence of the $K \nabla \nabla$ term, and therefore, the standard Schwinger-DeWitt algorithm used before cannot be applied here. The formalism for dealing with nonminimal operators is the generalized Schwinger-DeWitt technique of Barvinsky and Vilkovisky [29]. Nevertheless, this well elaborated technique of reducing expression (24) to the universal traces are not convenient here. In this situation, in order to work with such a complicated operator, we can follow the method developed in Ref. [16] for analogous calculations in the Lorentz/CPT violating electrodynamics.

The main idea is to introduce the inverse of the minimal operator $\hat{\mathbf{H}}_{m}^{-1}$ and make the transformation

$$
\begin{aligned}
\operatorname{Tr} \ln \hat{\mathbf{H}}= & \operatorname{Tr} \ln \left(\hat{\mathbf{H}}_{m}+\hat{\mathbf{H}}_{n m}\right) \\
= & \operatorname{Tr} \ln \hat{\mathbf{H}}_{m}+\operatorname{Tr} \ln \left(1+\hat{\mathbf{H}}_{m}^{-1} \cdot \hat{\mathbf{H}}_{n m}\right) \\
= & \operatorname{Tr} \ln \hat{\mathbf{H}}_{m}+\operatorname{Tr} \hat{\mathbf{H}}_{n m} \cdot \hat{\mathbf{H}}_{m}^{-1} \\
& -\frac{1}{2} \hat{\mathbf{H}}_{n m} \cdot \hat{\mathbf{H}}_{m}^{-1} \cdot \hat{\mathbf{H}}_{n m} \cdot \hat{\mathbf{H}}_{m}^{-1}+\cdots,
\end{aligned}
$$

where we have used the basic properties of the logarithm and performed its power series expansion. Now, the first term of last line of Eq. (25) contains only a minimal operator and can be directly calculated by the standard Schwinger-DeWitt technique [28], while the rest of the expression (25) contains nonlocal nonminimal structures, which can be, in principle, put into the form of the universal functional traces of Ref. [29].

Since the parameter $K^{\mu \nu}$ is dimensionless, it is possible to show, by power counting based arguments, that every term in the infinity series (25) gives contributions to the 
counterterms. The situation here is an analogue to the quantum gravity on a flat background, $g_{\mu \nu}=\eta_{\mu \nu}+h_{\mu \nu}$. Because the metric tensor is also dimensionless, there is in this theory, an infinite number of one-loop diagrams which are divergent. But, in the metric case, there is the principle of general covariance, allowing to transform all such infinite divergent contributions into a small number of covariant invariant expressions in terms of the curvature tensors $R_{\mu \nu \alpha \beta}, R_{\mu \nu}$, and $R$. In the case under consideration of the Lorentz/CPT violating scalar field, there is no principle allowing us to transform such an infinite number of counterterms (25) into specific invariants constructed from $K^{\mu \nu}$. Unfortunately, in this case, the series (25) must be truncated in some desired order. Since the Lorentz and $C P T$ violating parameters are assumed to be very small, the calculations for a general $\xi$ will be restricted to the first order in the symmetry-breaking terms. Then, the first order result of expression (25) is given by

$$
\operatorname{Tr} \ln \hat{\mathbf{H}}=\operatorname{Tr} \ln \hat{\mathbf{H}}_{m}+2 \operatorname{Tr} \hat{H}_{n m} \cdot \hat{H}_{0}^{-1},
$$

where

$$
\hat{H}_{0}=\square+m^{2}-\xi R
$$

is the standard bilinear operator for a scalar field nonminimally coupled with gravity and

$$
\hat{H}_{n m}=K^{\mu \nu} \nabla_{\mu} \nabla_{\nu} \text {. }
$$

Let us now consider the evaluation of the divergences contained in expression (26). The first term in this formula possesses only minimal differential operators, and it is possible to obtain the divergences, as before, by using the known formula of the Schwinger-DeWitt technique,

$$
\begin{aligned}
& \left.\frac{i}{2} \operatorname{Tr} \ln \hat{\mathbf{H}}_{m}\right|_{\text {div }} \\
& \quad=-\frac{\mu^{n-4}}{\epsilon} \int d^{n} x \sqrt{-g} \operatorname{tr}\left[\frac{\hat{\mathbf{1}}}{180}\left(R_{\mu \nu \alpha \beta}^{2}-R_{\alpha \beta}^{2}\right)+\frac{1}{2} \hat{\mathbf{P}}^{2}\right],
\end{aligned}
$$

where

$$
\hat{\mathbf{P}}=\hat{\mathbf{P}}_{0}-\nabla_{\mu} \hat{\mathbf{L}}^{\mu}+\cdots, \quad \text { with } \quad \hat{\mathbf{P}}_{0}=\hat{\mathbf{\Pi}}+\frac{\hat{\mathbf{1}}}{6} R .
$$

Then, up to the first order in the new parameters, we have

$$
\frac{1}{2} \operatorname{tr} \hat{\mathbf{P}}^{2}=\frac{1}{2} \operatorname{tr} \hat{\mathbf{P}}_{0}^{2}-\operatorname{tr} \hat{\mathbf{P}}_{0} \nabla_{\mu} \hat{\mathbf{L}}^{\mu}+\cdots
$$

and also,

$$
\begin{aligned}
\operatorname{tr} \hat{\mathbf{P}}_{0} \nabla_{\mu} \hat{\mathbf{L}}^{\mu} & =\left(\xi-\frac{1}{6}\right) R\left(\nabla_{\mu} \nabla_{\nu} K^{\mu \nu}+i \nabla_{\mu} k^{\mu}-i \nabla_{\mu} k^{\mu}\right) \\
& =\left(\xi-\frac{1}{6}\right) R \nabla_{\mu} \nabla_{\nu} K^{\mu \nu} .
\end{aligned}
$$

Formula (32) is the only source of contribution to divergences of the $C P T$-odd violating parameter $k^{\mu}(x)$. As explicitly shown, this parameter gives no contribution to vacuum renormalization. A similar situation occurs in the Lorentz and $C P T$-violating electrodynamics, where the $C P T$-odd $\left(k_{A F}\right)^{\mu}$ parameters also do not contribute to pure vacuum counterterms [14-16]. Just as in the QED case, we expect that the odd parameters may contribute to the interacting theory. In order to understand this, we can remember that in the scalar electrodynamics, e.g. there is a mixing between the gauge field $A_{\mu}(x)$ with the scalar field $\varphi(x)$ and the $C P T$-odd parameter $k_{\mu}(x)$ through the gauge covariant derivatives $D_{\mu}=\nabla_{\mu}+i e A_{\mu}$, which are present in the current term $j_{\mu}$.

Finally, by the use of Eqs. (31) and (32), formula (29) reduces to

$$
\begin{aligned}
& \left.\frac{i}{2} \operatorname{Tr} \ln \hat{\mathbf{H}}_{m}\right|_{\text {div }} \\
& =\Gamma_{\text {vac }}^{(1)}\left[g_{\mu \nu}\right]-\frac{\mu^{n-4}}{\epsilon} \int d^{n} x \sqrt{-g}\left(\xi-\frac{1}{6}\right) R \nabla_{\mu} \nabla_{\nu} K^{\mu \nu}+\cdots,
\end{aligned}
$$

where $\Gamma_{\text {vac }}^{(1)}\left[g_{\mu \nu}\right]$ is the divergent part of the metric dependent vacuum effective action of a nonminimally complex scalar field

$\Gamma_{\text {vac }}^{(1)}\left[g_{\mu \nu}\right]=-\frac{2 \mu^{n-4}}{\epsilon} \int d^{n} x \sqrt{-g}\left\{\frac{1}{180} R_{\mu \nu \alpha \beta}^{2}-\frac{1}{180} R_{\mu \nu}^{2}-\frac{1}{6}\left(\xi-\frac{1}{5}\right) \square R+\frac{1}{2}\left(\xi-\frac{1}{6}\right)^{2} R^{2}-m^{2}\left(\xi-\frac{1}{6}\right) R+\frac{m^{4}}{2}\right\}$.

For calculating the divergent part of the nonminimal piece of Eq. (26), we first need invert the operator $\hat{H}_{0}$ and find its nonlocal expression. Up to the background dimension of $1 / l^{4}$ (for introduction in this terminology, see Ref. [29]), the inverse operator can be expressed as

$$
\hat{H}_{0}^{-1}=\frac{1}{\square}+\left(\xi R-m^{2}\right) \frac{1}{\square^{2}}+\left(m^{4}-2 \xi m^{2} R+\xi^{2} R^{2}-\xi \square R\right) \frac{1}{\square^{3}}-2 \xi\left(\nabla^{\mu} R\right) \nabla_{\mu} \frac{1}{\square^{3}}+4 \xi\left(\nabla^{\mu} \nabla^{\nu} R\right) \nabla_{\mu} \nabla_{\nu} \frac{1}{\square^{4}}+\mathcal{O}\left(l^{-5}\right) .
$$

The higher background dimension, $\mathcal{O}\left(l^{-5}\right)$ terms, can be safely omitted here because they do not contribute to divergences [29]. Using Eq. (28), one can obtain the relation 


$$
\begin{aligned}
\operatorname{Tr} \hat{H}_{n m} \cdot \hat{H}_{0}^{-1}= & K^{\mu \nu} \nabla_{\mu} \nabla_{\nu} \frac{1}{\square}+\xi K^{\mu \nu}\left(\nabla_{\mu} \nabla_{\nu} R\right) \frac{1}{\square^{2}}+\left(\xi R-m^{2}\right) K^{\mu \nu} \nabla_{\mu} \nabla_{\nu} \frac{1}{\square^{2}} \\
& -4 \xi K^{\mu \alpha}\left(\nabla_{\alpha} \nabla^{\nu} R\right) \nabla_{\mu} \nabla_{\nu} \frac{1}{\square^{3}}+\left(m^{4}-2 m^{2} \xi R+\xi^{2} R^{2}-\xi \square R\right) K^{\mu \nu} \nabla_{\mu} \nabla_{\nu} \frac{1}{\square^{3}} \\
& +4 \xi\left(\nabla^{\mu} \nabla^{\nu} R\right) K^{\alpha \beta} \nabla_{\alpha} \nabla_{\beta} \nabla_{\mu} \nabla_{\nu} \frac{1}{\square^{4}}+\mathcal{O}\left(l^{-5}\right) .
\end{aligned}
$$

Once more, in the last formula, we do not write explicitly the $\mathcal{O}\left(l^{-5}\right)$ structures and also the functional traces with dimensionality $l^{-3}$, because they are irrelevant to the divergences.
Expression (36) is already in the form that allows us to apply the tables of universal functional traces of Ref. [29]. The calculation is straightforward, and the intermediary results are shown in Appendix A. The final result has the form

$$
\begin{aligned}
\left.\frac{i}{2} \operatorname{Tr} \hat{H}_{n m} \cdot \hat{H}_{0}^{-1}\right|_{\mathrm{div}}= & -\frac{\mu^{n-4}}{\epsilon} \int d^{n} x \sqrt{-g}\left\{\frac{1}{60} R_{\mu \nu} \square K^{\mu \nu}+\left(\frac{1}{20}-\frac{1}{3} \xi\right) R \nabla_{\mu} \nabla_{\nu} K^{\mu \nu}+\frac{1}{90} K^{\mu \nu} R^{\alpha \beta} R_{\alpha \mu \beta \nu}\right. \\
& +\frac{1}{90} K^{\mu \nu} R_{\mu \rho \alpha \beta} R_{\nu}^{\rho \alpha \beta}-\frac{1}{45} K^{\mu \nu} R_{\mu \alpha} R_{\nu}^{\alpha}-\frac{1}{6}\left(\xi-\frac{1}{6}\right) K^{\mu \nu} R R_{\mu \nu}+\frac{m^{2}}{6} K^{\mu \nu} R_{\mu \nu} \\
& \left.-\frac{K}{2}\left[\frac{1}{180} R_{\mu \nu \alpha \beta}^{2}-\frac{1}{180} R_{\mu \nu}^{2}-\frac{1}{6}\left(\xi-\frac{1}{5}\right) \square R+\frac{1}{2}\left(\xi-\frac{1}{6}\right)^{2} R^{2}-m^{2}\left(\xi-\frac{1}{6}\right) R+\frac{m^{4}}{2}\right]\right\} .
\end{aligned}
$$

Finally, from Eqs. (24), (26), (33), and (37), we arrive at the result for the one-loop divergences in the first order in the Lorentz violating parameter

$$
\begin{aligned}
\Gamma_{\mathrm{div}}^{(1)}= & -\frac{2 \mu^{n-4}}{\epsilon} \int d^{n} x \sqrt{-g}\left\{\frac{1}{60} R_{\mu \nu} \square K^{\mu \nu}+\frac{1}{6}\left(\xi-\frac{1}{5}\right) R \nabla_{\mu} \nabla_{\nu} K^{\mu \nu}+\frac{1}{90} K^{\mu \nu} R^{\alpha \beta} R_{\alpha \mu \beta \nu}\right. \\
& +\frac{1}{90} K^{\mu \nu} R_{\mu \rho \alpha \beta} R_{\nu}^{\rho \alpha \beta}-\frac{1}{45} K^{\mu \nu} R_{\mu \alpha} R_{\nu}^{\alpha}-\frac{1}{6}\left(\xi-\frac{1}{6}\right) K^{\mu \nu} R R_{\mu \nu} \\
& \left.-\frac{K}{2}\left[\frac{1}{180} R_{\mu \nu \alpha \beta}^{2}-\frac{1}{180} R_{\mu \nu}^{2}-\frac{1}{6}\left(\xi-\frac{1}{5}\right) \square R+\frac{1}{2}\left(\xi-\frac{1}{6}\right)^{2} R^{2}-m^{2}\left(\xi-\frac{1}{6}\right) R+\frac{m^{4}}{2}+\frac{1}{6} m^{2} K^{\mu \nu} R_{\mu \nu}\right]\right\}+\Gamma_{\text {vac }}^{(1)}\left[g_{\mu \nu}\right] .
\end{aligned}
$$

Let us start the analysis of our result. First of all, we can verify that in the $\xi=0$ limit, we arrive exactly at the same result for the first order one-loop divergences in the minimal case Eq. (17) obtained by the previously calculation method. ${ }^{3}$ Second, the vacuum part (34) has the well-known standard form of the divergences in curved spacetime for the massive scalar field theory nonminimally interacting with gravity. This is perfectly consistent with the general features of renormalization in curved spacetime, because the semiclassical renormalizable theory always includes higher derivative terms in the gravitational sector (see, e.g. [13,19]). In our case, this also means that the renormalization of the nonviolating sector is performed independently on the external Lorentz/CPT symmetrybreaking fields. In the case when some of the violating fields are present, the consistent form of the vacuum action

\footnotetext{
${ }^{3}$ The extra 2 factor is because the theory of $N$ complex scalar fields can also be written in terms of a model with $2 N$ real scalar fields.
}

becomes much more complicated and involves the dependence on these extra fields. Our one-loop calculations show which terms can emerge as counterterms in the scalar field case. Therefore, the minimal set of structures which are requested by renormalizability in the gravitational action can be expressed as

$$
S_{\text {grav }}=\int d^{4} x \sqrt{-g}\left\{v(x)+u(x) R+s^{\mu \nu}(x) R_{\mu \nu}\right\}+S_{\mathrm{HD}},
$$

where the last part $S_{\mathrm{HD}}$ represents the generalized higher derivative term

$$
\begin{aligned}
S_{\mathrm{HD}}= & \int d^{4} x \sqrt{-g}\left\{\Phi_{1}(x) R_{\mu \nu \alpha \beta}^{2}+\Phi_{2}(x) R_{\mu \nu}^{2}\right. \\
& +\Phi_{3}(x) \square R+\Phi_{4}(x) R^{2}+\zeta_{1}^{\mu \nu}(x) R_{\mu \rho \alpha \beta} R_{\nu}^{\rho \alpha \beta} \\
& \left.+\zeta_{2}^{\mu \nu}(x) R^{\alpha \beta} R_{\mu \alpha \nu \beta}+\zeta_{3}^{\mu \nu}(x) R_{\mu \rho} R_{\nu}^{\rho}+\zeta_{4}^{\mu \nu}(x) R R_{\mu \nu}\right\} .
\end{aligned}
$$


Let us notice that the terms $t^{\mu \nu \alpha \beta}(x) R_{\mu \nu \alpha \beta}$, $\eta_{1}^{\mu \nu \alpha \beta}(x) R_{\rho \omega \mu \nu} R_{. . \alpha \beta}^{\rho \omega}, \eta_{2}^{\mu \nu \alpha \beta}(x) R_{\mu \rho} R_{. \nu \alpha \beta}^{\rho}, \eta_{3}^{\mu \nu \alpha \beta}(x) R R_{\mu \nu \alpha \beta}$, and $\eta_{4}^{\mu \nu \alpha \beta}(x) R_{\mu \alpha} R_{\nu \beta}$ which are necessary for the renormalization of Lorentz violating electrodynamics do not appear here in the scalar field case. Indeed this is expected due to the different tensorial properties of the symmetry-breaking fields in these two theories. Of course, this is also for the reason that we work only in first order in the Lorentz/CPT violating parameters, and we expect that such structures will emerge in higher order calculations. In principle, the nonlinear terms can also be derived from the general expansion (25); however, the derivation of such complicated functional traces will require significant efforts.

The most remarkable aspect of the result (38) is that the cosmological constantlike divergence $m^{4}$ appears multiplied by a coefficient $K$ which may be coordinate dependent. This means that in the theory where the Lorentz-violating parameter $K^{\mu \nu}$ is not a constant, the cosmological constant cannot be constant, but should have some coordinate dependence. It would be certainly interesting to derive the upper bound for the time dependence of $K(x)$ from laboratory experiments and compare it to the bounds for variable vacuum energy density in cosmology. Also, many of the other new structures present in Eqs. (39) and (40) can be implied in new gravitational physical effects. The investigation of the possible phenomenological manifestations of terms linear in curvature was performed in Ref. [42] on the basis of PPN formalism, and recently, an extensive systematic analysis of the Lorentz violating higher derivatives terms has been started in [43], also in the weak gravitational field approximation. According to [43], the presence of higher derivative violating terms leads to a modified Poisson equation for the gravitational potential $\varphi_{g}(r)$ in the form

$$
\Delta \varphi_{g}(r)=-4 \pi G \rho(r)+\left(k_{\text {eff }}^{i j l k}\right) \partial_{i} \partial_{j} \partial_{l} \partial_{k} \varphi_{g}(r),
$$

which implies diverse new phenomenological consequences. In formula (41), the violating parameter $k_{\mathrm{eff}}^{i j k}$ is constructed on the basis of the symmetry-breaking fields present in the higher derivative sector of gravitational action. Additionally, in Ref. [44], the role of some Lorentz violating higher derivative terms was analyzed in the quantum gravity framework. Since action (39) is requested by the renormalization of the Lorentz/CPT violating SME matter sector, the detailed analysis of all the contained structures deserve special attention in both classical and quantum levels.

\section{LOCAL CONFORMAL SYMMETRY AND CONFORMAL ANOMALY}

It is pretty well known that the classical action of a free scalar field theory in curved spacetime is invariant, in the $m=0$ and $\xi=1 / 6$ limit, under the following transformations:

$$
\begin{aligned}
g_{\mu \nu} \rightarrow g_{\mu \nu}^{\prime} & =g_{\mu \nu} \cdot e^{2 \sigma(x)} \quad \text { and } \\
\varphi \rightarrow \varphi^{\prime} & =\varphi \cdot e^{-\sigma(x)} .
\end{aligned}
$$

The formula (42) is called the local conformal transformation, and the corresponding action invariance is known as local conformal symmetry. The form of the Noether identity corresponding to this symmetry, in the on shell limit, ${ }^{4}$ is

$$
2 g_{\mu \nu} \frac{\delta S}{\delta g_{\mu \nu}}=0,
$$

which is interpreted as the vanishing trace of the energymomentum tensor $T_{\mu}^{\mu}=0$. It is very important to note that the classical action of a scalar field with Lorentz and CPT symmetry breaking terms (1) also possesses local conformal invariance in the aforementioned limit, if we allow the Lorentz and/or $C P T$-violating parameters to transform according to

$$
\begin{aligned}
K^{\mu \nu} \rightarrow K^{\prime \mu \nu} & =K^{\mu \nu} \cdot e^{-2 \sigma(x)}, \\
k^{\mu} \rightarrow k^{\prime \mu} & =k^{\mu} \cdot e^{-2 \sigma(x)} .
\end{aligned}
$$

The breaking of Eq. (43) occurs only at quantum level because of the renormalization procedure. Such phenomenon is known as conformal anomaly or, simply, trace anomaly [22]. At a quantum level, the classical action of vacuum has to be replaced by the renormalized effective action

$$
\Gamma_{R}=S+\Gamma^{(1)}+\Delta S,
$$

where $S$ is the classical action, $\Gamma^{(1)}$ is the bare one-loop effective action, and $\Delta S$ is a local counterterm which is requested to cancel the divergent part of $\Gamma^{(1)}$. The counterterm $\Delta S$ is the only source of nonconformal invariance of the effective action, because both the classical action and direct quantum contribution are conformal invariant [13]. Thus, the expectation value of the trace $\left\langle T_{\mu}^{\mu}\right\rangle$ differs from zero and can be expressed by

$\left\langle T_{\mu}^{\mu}\right\rangle=-\left.\frac{2}{\sqrt{-g}} g_{\mu \nu} \frac{\delta \Gamma_{R}}{\delta g_{\mu \nu}}\right|_{n=4}=-\left.\frac{2}{\sqrt{-g}} g_{\mu \nu} \frac{\delta \Delta S}{\delta g_{\mu \nu}}\right|_{n=4}$.

Let us stress that the anomaly induced EA is nonlocal, as we will see later on in Eq. (65). However, the anomaly itself can be derived from the local counterterm, as explained in [45] and recently, in [23,46].

\footnotetext{
${ }^{4}$ For Eq. (43) to be valid in the Lorentz/CPT violating theories, the symmetry-breaking parameters must obey their own dynamical equations. As discussed in [11], this can be achieved if the violating fields originates from some spontaneous symmetry breaking mechanism.
} 
The form of the counterterm $\Delta S=-\Gamma_{\text {div }}^{(1)}$ for the conformal version of theory (1) can be obtained from Eq. (38). The answer is

$$
\Delta S=\frac{2 \mu^{n-4}}{\epsilon} \int d^{n} x \sqrt{-g} L\left(g_{\mu \nu}, K_{\mu \nu}\right)-\Gamma_{\text {vac }}^{(1)}\left[g_{\mu \nu}\right],
$$

where

$$
\begin{aligned}
L\left(g_{\mu \nu}, K_{\mu \nu}\right)= & \frac{1}{60} R_{\mu \nu} \square K^{\mu \nu}-\frac{1}{180} R \nabla_{\mu} \nabla_{\nu} K^{\mu \nu} \\
& +\frac{1}{90} K^{\mu \nu} R^{\alpha \beta} R_{\alpha \mu \beta \nu}+\frac{1}{90} K^{\mu \nu} R_{\mu \rho \alpha \beta} R_{\nu}^{\rho \alpha \beta} \\
& -\frac{1}{45} K^{\mu \nu} R_{\mu \alpha} R_{\nu}^{\alpha}-\frac{K}{2}\left(\frac{1}{180} R_{\mu \nu \alpha \beta}^{2}-\frac{1}{180} R_{\mu \nu}^{2}\right. \\
& \left.+\frac{1}{180} \square R\right)
\end{aligned}
$$

and

$$
\Gamma_{\text {vac }}^{(1)}\left[g_{\mu \nu}\right]=-\frac{2 \mu^{n-4}}{\epsilon} \int d^{n} x \sqrt{-g}\left\{\frac{1}{120} C^{2}-\frac{1}{360} E+\frac{1}{180} \square R\right\} .
$$

In the above formula, $C^{2}=C_{\mu \nu \alpha \beta} C^{\mu \nu \alpha \beta}=R_{\mu \nu \alpha \beta}^{2}-2 R_{\mu \nu}^{2}+$ $\frac{1}{3} R^{2}$ is the square of Weyl tensor and $E=R_{\mu \nu \alpha \beta}^{2}-4 R_{\mu \nu}^{2}+$ $R^{2}$ is the integrand of the Gauss-Bonnet topological term (Euler density in $n=4$ ).

The calculation of the expression (46) with the counterterm (47) can be done by many different ways $[19,45,47-$ 50]. Following Ref. [23], the simplest one is by using the conformal parametrization of the metric,

$$
g_{\mu \nu}=g_{\mu \nu}^{\prime} \cdot e^{2 \sigma(x)},
$$

and by the direct application of the chain rule

$-\frac{2}{\sqrt{-g}} g_{\mu \nu} \frac{\delta A\left[g_{\mu \nu}\right]}{\delta g_{\mu \nu}}=-\left.\frac{1}{\sqrt{-g^{\prime}}} e^{-4 n \sigma} \frac{\delta A\left[g_{\mu \nu}^{\prime} e^{2 \sigma}\right]}{\delta \sigma}\right|_{g_{\mu \nu}^{\prime} \rightarrow g_{\mu \nu}, \sigma \rightarrow 0}$,

which is valid for any functional $A=A\left[g_{\mu \nu}\right]$. This procedure can be seen as a purely technical one, and the form of the metric (50) is discarded after the anomaly derivation.

One of the key parts of this general procedure are the conformal transformation rules of each quantity present in Eq. (47). Besides the pure curvature terms, whose transformation rules can be found elsewhere [51], we also need the transformation rule for the new Lorentz violating term (48). In fact, in the four dimensional spacetime, this term is the conformal invariant

$$
\int d^{4} x \sqrt{-g^{\prime}} L\left(g_{\mu \nu}^{\prime}, K_{\mu \nu}^{\prime}\right)=\int d^{4} x \sqrt{-g} L\left(g_{\mu \nu}, K_{\mu \nu}\right) .
$$

For the convenience of the reader, we present the proof of Eq. (52) in Appendix B. Indeed, the conformal symmetry (52) of the quantum correction in the four dimensional spacetime limit is expected to hold for conformal theories based on general standard arguments (see, e.g. [13]); thus, the direct algebraic proof of Eq. (52) can also be seen as a test of the verification of the cumbersome calculations which led to the answer (38). On the top of that, formula (52) also implies that in the generic spacetime with $n$ dimensions, the generalized $n$-dimensional form of Eq. (52) gains a global $e^{(n-4) \sigma}$ multiplicative factor, besides some possible extra terms with derivatives of $\sigma(x)$. All other expressions of our interest have the same general structure with the multiplicative exponential factor, and the nonexponential terms are irrelevant due to the limit procedure in Eq. (51). Consequently, the application of the identity (51) becomes simple. Then, one can find the final answer for the conformal anomaly,

$$
\left\langle T_{\mu}^{\mu}\right\rangle=-\left[w C^{2}+b E+c \square R+2 L\left(g_{\mu \nu}, K_{\mu \nu}\right)\right],
$$

where the parameters $w, b, c$ are, in the complex scalar field case,

$$
(w, b, c)=\frac{2}{(4 \pi)^{2}}\left(\frac{1}{120},-\frac{1}{360}, \frac{1}{180}\right) .
$$

In the case of local conformal invariance, there is always a well-known ambiguity in the value of the $c$ parameter $[19,22,49]$. In a simplified way, the qualitative net result is that this ambiguity is always equivalent to the freedom to add the local $R^{2}$ term to the classical action, since

$$
-\frac{2}{\sqrt{-g}} g_{\mu \nu} \frac{\delta}{\delta g_{\mu \nu}} \int d^{4} x \sqrt{-g} R^{2}=12 \square R .
$$

For more details in this subject, the reader is referred to [49], where this issue was addressed with all technicalities.

\section{ANOMALY INDUCED EFFECTIVE ACTION}

One can use the conformal anomaly (53) to construct a differential equation for the one-loop effective action

$$
\frac{2}{\sqrt{-g}} g_{\mu \nu} \frac{\delta \Gamma_{\text {ind }}}{\delta g_{\mu \nu}}=w C^{2}+b E+c \square R+2 L .
$$

The solution of Eq. (56) is known as anomaly induced effective action. The integration of a conformal anomaly is by the technical side not very difficult in the usual theory without the Lorentz violating term [24], and it remains identically simple when this term is present [16]. The reason is because the new violating term (48) possesses the same conformal properties of the square of Weyl tensor, which makes its inclusion a very simple exercise.

The anomaly induced effective action can be presented in the simplest way by a noncovariant form or in a more complicated one which is covariant and nonlocal. Additionally, by the introduction of auxiliary fields, it can 
also be cast into a dynamically equivalent local and covariant form. Let us start from the simplest case and parametrize the metric tensor as in (50), separating its conformal factor $\sigma(x)$. After that, we can rewrite Eq. (56) using the relation (51) and the conformal transformation rules [51]

$$
\begin{gathered}
\sqrt{-g} C^{2}=\sqrt{-g^{\prime}} C^{2}, \\
\sqrt{-g}\left(E+\frac{2}{3} \square R\right)=\sqrt{-g^{\prime}}\left(E^{\prime}+\frac{2}{3} \square^{\prime} R^{\prime}+4 \Delta_{4}^{\prime} \sigma\right), \\
\sqrt{-g} \Delta_{4}=\sqrt{-g^{\prime}} \Delta_{4}^{\prime}
\end{gathered}
$$

together with the Lorentz violating term transformation, Eq. (52). Here and below, the quantities with primes are constructed using only the metric $g_{\mu \nu}^{\prime}$. In particular, in the above formula, $\Delta_{4}$ is the Paneitz operator [52]

$$
\Delta_{4}=\square^{2}+2 R^{\mu \nu} \nabla_{\mu} \nabla_{\nu}-\frac{2}{3} R \square+\frac{1}{3}\left(\nabla^{\mu} R\right) \nabla_{\mu},
$$

which is a covariant, fourth derivative, self-adjoint, and conformal invariant operator when acting on dimensionless scalar fields.

After the described procedure is a completed formula (56) becomes very simple, and integration in the $\sigma$ variable is straightforward. The solution for the effective action is

$$
\begin{aligned}
\Gamma_{\text {ind }}= & S_{c}\left[g_{\mu \nu}^{\prime}, K_{\mu \nu}^{\prime}\right]+\int d^{4} x \sqrt{-g^{\prime}}\left\{w \sigma C^{\prime 2}+b \sigma\left(E^{\prime}-\frac{2}{3} \square^{\prime} R^{\prime}\right)\right. \\
& +2 b \sigma \Delta_{4}^{\prime} \sigma+2 \sigma L\left(g_{\mu \nu}^{\prime}, K_{\mu \nu}^{\prime}\right) \\
& \left.-\frac{3 c+2 b}{36}\left[R^{\prime}-6\left(\nabla^{\prime} \sigma\right)^{2}-6 \square^{\prime} \sigma\right]^{2}\right\},
\end{aligned}
$$

where $S_{c}=S_{c}\left[g_{\mu \nu}, K_{\mu \nu}\right]$ is an unknown conformal invariant functional, which serves as an integration constant for Eq. (56) and cannot be uniquely defined in the present scheme. In the theories where the unique background field is metric, due to the conformal invariance, this term is irrelevant for the dynamics of the conformal factor of the metric, e.g. in the cosmological case with the FriedmannLemaître-Robertson-Walker (FLRW) metric. At the same time, even in some more complicated cases, the conformal invariant term can be ignored as a good approximation. The reason is that the $S_{c}$ may contain only subleading quantum corrections, while the rest of the action (61) has the leading logarithm corrections [46] with full information about the ultraviolet limit of theory. This affirmation is based, in particular, on the results obtained without this conformal term which provide a very nice match with the outputs of other methods, as in the gravitational waves [53-55] and black hole [56] cases.

In the present case, with the extra nonmetric background fields, the irrelevance of the conformal term does not hold even for the cosmological background. In this sense, the anomaly induced effective action is not unique defined and this can, in principle, affect some physical applications. It would be interesting to analyze the relevance of this term within another approach, such as the curvature expansion [50], which enables one to obtain information about the conformal invariant term $S_{c}$.

The solution (61) is noncovariant, in the sense that it is not written in terms of the original metric $g_{\mu \nu}$. In order to obtain the covariant solution, we can use the following formula which is true for any conformal functional $A\left[g_{\mu \nu}\right]=A\left[g_{\mu \nu}^{\prime}\right]$ :

$$
\begin{aligned}
2 g_{\mu \nu}(y) \frac{\delta}{\delta g_{\mu \nu}(y)} \int d^{4} x \sqrt{-g(x)} A\left(E-\frac{2}{3} \square R\right)_{x} & =\left.\frac{\delta}{\delta \sigma(y)} \int d^{4} x \sqrt{-g^{\prime}(x)} A^{\prime}\left(E^{\prime}-\frac{2}{3} \square^{\prime} R^{\prime}+4 \Delta_{4}^{\prime} \sigma\right)_{x}\right|_{g_{\mu \nu}^{\prime} \rightarrow g_{\mu \nu}, \sigma \rightarrow 0} \\
& =4 \sqrt{-g^{\prime}(y)} \Delta_{4}^{\prime} A^{\prime}=4 \sqrt{-g(y)} \Delta_{4} A .
\end{aligned}
$$

In the above equation we have used the identity (51) together with the transformation rule (58). Introducing the Green function for the Paneitz operator,

$$
\sqrt{-g(x)} \Delta_{4, x} G(x, y)=\delta^{4}(x-y)
$$

and by means of the relation (62), solving Eq. (56) becomes direct. For example, for the Weyl squared term

$$
\begin{aligned}
2 g_{\mu \nu} & \frac{\delta}{\delta g_{\mu \nu}(y)} \int d^{4} x \sqrt{-g(x)} \int d^{4} y \sqrt{-g(y)} C^{2}(x) G(x, y)\left(E-\frac{2}{3} \square R\right)_{y} \\
& =4 \int d^{4} x \sqrt{-g(y)} \Delta_{4, y} G(x, y) C^{2}(x)=4 C^{2}(y) .
\end{aligned}
$$

Using an analogous consideration for the other terms in (56) and by means of the formula (55), we arrive at the solution

$$
\Gamma_{\text {ind }}=S_{c}+\Gamma_{w}+\Gamma_{b}+\Gamma_{c}
$$


where

$$
\begin{aligned}
& \Gamma_{w}=\frac{1}{4} \int d^{4} x \sqrt{-g(x)} \int d^{4} y \sqrt{-g(y)}\left(w C^{2}+2 L\right)_{x} G(x, y)\left(E-\frac{2}{3} \square R\right)_{y}, \\
& \Gamma_{b}=\frac{b}{8} \int d^{4} x \sqrt{-g(x)} \int d^{4} y \sqrt{-g(y)}\left(E-\frac{2}{3} \square R\right)_{x} G(x, y)\left(E-\frac{2}{3} \square R\right)_{y}
\end{aligned}
$$

and

$$
\Gamma_{c}=-\frac{3 c+2 b}{36} \int d^{4} x \sqrt{-g(x)} R^{2}(x) .
$$

One can note that the Lorentz violating terms show up only in the first nonlocal term, Eq. (66).

At the next stage, the nonlocal expressions for the anomaly induced EA can be presented in a local form through the introduction of two auxiliary scalar fields $\phi(x)$ and $\psi(x)$ [57] (the simpler one scalar form was known from much earlier, see [24]). This procedure was discussed in detail in Ref. [57] and revised in [23,46], so let us give just a final result for the local form of the anomaly induced effective action,

$$
\begin{aligned}
\Gamma_{\text {ind }}= & S_{c}\left[g_{\mu \nu}, K_{\mu \nu}\right]-\frac{3 c+2 b}{36} \int d^{4} x \sqrt{-g(x)} R^{2}(x) \\
& +\int d^{4} x \sqrt{-g(x)}\left\{\frac{1}{2} \phi \Delta_{4} \phi\right. \\
& -\frac{1}{2} \psi \Delta_{4} \psi+\phi\left[k_{1}\left(C^{2}+\frac{2}{w} L\right)+k_{2}\left(E-\frac{2}{3} \square R\right)\right] \\
& \left.+l_{1} \psi\left(C^{2}+\frac{2}{w} L\right)\right\},
\end{aligned}
$$

where

$$
k_{1}=-l_{1}=-\frac{w}{2 \sqrt{-b}} \quad \text { and } \quad k_{2}=\frac{\sqrt{-b}}{2} .
$$

At the classical level, the local covariant form (69) is dynamically equivalent to the nonlocal covariant one (65), which means that after solving the field equations for the fields $\phi(x)$ and $\psi(x)$ and plugging back these solutions in to action (69), we come back to the previous formula (65). The use of the local covariant form with auxiliary scalars is advantageous because the initial value problem for these fields are equivalent to the boundary conditions for the two Green functions present in the nonlocal covariant form (65). By this reason, Eq. (69) is the most useful one for dealing with Hawking radiation from black holes $[56,58]$ or exploring the dynamics of gravitational waves on cosmological background [54]. Also, the relevance to have two auxiliary fields instead of a single one field has been addressed in detail in Refs. [56,57].
The actions (61), (65), (69) represent the final product of the conformal anomaly integration. They correspond to the quantum correction to the classical gravitational action. In comparison with the previous standard case known in the literature, those formulas have extra Lorentz violating terms coming from the scalar field contribution. All information about the symmetry-breaking parameters is included in the $L\left(g_{\mu \nu}, K_{\mu \nu}\right)$ function. An analogous situation was found in Ref. [16] in the Lorentz/CPT violating electrodynamics. In that case, the full vector field contribution was contained in a single function involving the $C P T$-even violating parameter $k_{F}^{\mu \nu \alpha \beta}$ and curvature tensors. Additionally, in that work, it has been shown that the new term does not affect the dynamics for the FLRW metrics with generic spatial curvature values $k=0, \pm 1$. This negative result concerning the effect of the new term in the homogeneous and isotropic spacetime is expected, since the violating fields defines a preferable direction in that background. Therefore, this fact can also be seen as an additional test for our huge algebraic calculations. Indeed, in our case, it is not difficult to show that $L\left(g_{\mu \nu}^{\mathrm{FLRW}}, K_{\mu \nu}\right)=0$. Besides that, one can expect that the new terms can cause some modifications in the equations for cosmic perturbations during the inflationary epoch and especially, for gravitational waves. The study of gravitational waves in the anomaly induced effective action formalism has been done systematically in Ref. [54], and its generalization with the presence of the extra Lorentz violating term would be certainly a potentially interesting problem. One can expect relevant different contributions which can lead to some new constraints on the symmetry-breaking parameter $K^{\mu \nu}$. Regardless of the serious technical difficulties of this program, it does not look unreliable in practice.

\section{CONCLUSIONS}

Let us summarize the results obtained. We have calculated the vacuum one-loop divergences for the Lorentz/CPT violating scalar field theory in a curved background. The symmetry-violating parameters were treated as fields, rather than constants. The practical calculations have been performed for scalars with a minimal and nonminimal interaction with gravity by the application of functional methods and the Schwinger-DeWitt technique [28,29]. For minimal real scalars, the solution for the one-loop counterterms was 
found in a closed form, while for the nonminimal complex scalar field, the solution has been obtained in the first order in the small symmetry-breaking parameters. It turns out that the $C P T$-odd violating fields do not contribute for the vacuum divergences at that order. All contribution to the renormalization of the vacuum comes from the dimensionless parameter $K^{\mu \nu}$, an analogous situation to what happens in Lorentz violating $\mathrm{QED}$, where only the $C P T$-even parameter $k_{F}^{\mu \nu \alpha \beta}$ contributes [14-16]. At same time, we expect that the odd parameter becomes relevant in the interacting theory and/or in higher orders in Lorentz/CPT symmetry breaking fields. Also, the minimal form of the gravitational action requested by the renormalization of the violating scalar field theory in curved spacetime was established based on the previous one-loop calculations. In particular, the effect of some Lorentz violating gravitational terms, which are necessary at a semiclassical quantum level, were already discussed in the short range gravity limit $[42,43]$ and in the quantum gravity [44]. At the next stage, a similar analysis for the other missing new terms in Eq. (39) and those coming from the photon sector [16] would be a very interesting exercise, and we hope that with such analysis some new bounds on the gravitational Lorentz violating parameters will be established.

The derivation of one-loop divergences for scalar fields with a nonminimal gravitational coupling also opens the way to study the conformal anomaly and anomaly induced effective action of gravity, whose derivations did not bring up serious obstacles. The anomaly integration proceeds with minimal changes compared to the known procedure, since the new Lorentz violating term is conformal. At the one-loop level, the anomaly is given by an algebraic sum of the contributions of the massless conformal invariant fields of spins $0,1 / 2,1$. The expression obtained, Eq. (69), represents the scalar field contribution for gravitational effective action, and together with the photon part [16] must be completed with the fermionic contribution, which we are planning to present elsewhere. After that, the use of the corresponding gravitational effective action in searching for the Lorentz violation in the anisotropies of cosmic microwave radiation, coming from the cosmic perturbations in the early Universe, would represent a promising area for application of our results.

\section{ACKNOWLEDGMENTS}

The author wishes to acknowledge Coordenação de Aperfeiçoamento de Pessoal de Nível Superior (CAPES) for the support through the PNPD program. The author is also grateful to Ilya L. Shapiro and Breno Loureiro Giacchini for useful discussions.

\section{APPENDIX A: INTERMEDIARY UNIVERSAL FUNCTIONAL TRACES RESULTS}

To obtain the divergent part of the nonminimal piece of a one-loop effective action (36), we shall use the table universal functional traces which are an important part of the generalized Schwinger-DeWitt technique. The mentioned table correspond to the formulas (4.53) up to (4.61) of Ref. [29] (note that here we use opposite sign notations). Using these formulas, the divergences in each term of Eq. (36) can be directly calculated. After some algebra, we obtain

$$
\begin{gathered}
\left.\operatorname{Tr} K^{\mu \nu} \nabla_{\mu} \nabla_{\nu} \frac{1}{\square}\right|_{\text {div }}=\frac{2 i \mu^{n-4}}{\epsilon} \int d^{n} x \sqrt{-g}\left\{K^{\mu \nu}\left(\frac{1}{90} R^{\alpha \beta} R_{\alpha \mu \beta \nu}+\frac{1}{90} R_{\alpha \beta \rho \mu} R_{\ldots \nu}^{\alpha \beta \rho}-\frac{1}{45} R_{\mu \alpha} R_{\nu}^{\alpha}+\frac{1}{36} R R_{\mu \nu}+\frac{1}{60} \square R_{\mu \nu}+\frac{1}{20} \nabla_{\mu} \nabla_{\nu} R\right)\right. \\
\left.-\frac{K}{2}\left(\frac{1}{180} R_{\mu \nu \alpha \beta}^{2}-\frac{1}{180} R_{\alpha \beta}^{2}+\frac{1}{72} R^{2}+\frac{1}{30} \square R\right)\right\}, \\
\left.\operatorname{Tr}\left(\xi R-m^{2}\right) K^{\mu \nu} \nabla_{\mu} \nabla_{\nu} \frac{1}{\square^{2}}\right|_{\text {div }}=\frac{i \mu^{n-4}}{3 \epsilon} \int d^{n} x \sqrt{-g}\left\{\frac{1}{2} K \xi R^{2}-\xi K^{\mu \nu} R R_{\mu \nu}+m^{2} K^{\mu \nu} R_{\mu \nu}-\frac{m^{2}}{2} K R\right\}, \\
\begin{array}{c}
\left.\operatorname{Tr}\left(m^{4}-2 m^{2} \xi R+\xi^{2} R^{2}-\xi \square R\right) K^{\mu \nu} \nabla_{\mu} \nabla_{\nu} \frac{1}{\square^{3}}\right|_{\text {div }}=-\frac{i \mu^{n-4}}{2 \epsilon} \int d^{n} x \sqrt{-g}\left(m^{4}-2 m^{2} \xi R+\xi^{2} R^{2}-\xi \square R\right) K, \\
\left.4 \operatorname{Tr} \xi\left(\nabla^{\mu} \nabla^{\nu} R\right) K^{\alpha \beta} \nabla_{\alpha} \nabla_{\beta} \nabla_{\mu} \nabla_{\nu} \frac{1}{\square^{4}}\right|_{\text {div }}=-\frac{i \mu^{n-4}}{3 \epsilon} \int d^{n} x \sqrt{-g}\left\{\xi K \square R+2 \xi K^{\mu \nu} \nabla_{\mu} \nabla_{\nu} R\right\}, \\
\left.\operatorname{Tr} \xi K^{\mu \nu}\left(\nabla_{\mu} \nabla_{\nu} R\right) \frac{1}{\square^{2}}\right|_{\text {div }}=-\frac{2 i \mu^{n-4}}{\epsilon} \int d^{n} x \sqrt{-g} \xi K^{\mu \nu} \nabla_{\mu} \nabla_{\nu} R, \\
-\left.4 \operatorname{Tr} \xi K^{\mu \alpha}\left(\nabla_{\alpha} \nabla^{\nu} R\right) \nabla_{\mu} \nabla_{\nu} \frac{1}{\square^{3}}\right|_{\text {div }}=\frac{2 i \mu^{n-4}}{\epsilon} \int d^{n} x \sqrt{-g} \xi K^{\mu \nu} \nabla_{\mu} \nabla_{\nu} R .
\end{array}
\end{gathered}
$$

By using Eqs. (36) and relations (A1)-(A6), one can obtain the result (37). 


\section{APPENDIX B: PROOF OF CONFORMAL INVARIANCE OF THE LORENTZ VIOLATING $L$ TERM}

Let us present here the proof of conformal invariance (52). From the technical side, this is not a trivial task since the expression (48) is quite complicated; then we are going to expose some details concerning the needed conformal transformation rules. Since the conformal group is a one-parameter Lie group, one can restrict our considerations to the infinitesimal version of transformation (42). Disregarding the higher orders in $\sigma$ and superficial terms, after some tedious algebra, we arrive at the following transformation rules for each term present in (48):

$$
\begin{gathered}
\left(\sqrt{-g} K^{\mu \nu} R_{\mu \rho \alpha \beta} R_{\nu}^{\rho \alpha \beta}\right)^{\prime}=\sqrt{-g}\left[K^{\mu \nu} R_{\mu \rho \alpha \beta} R_{\nu}^{\rho \alpha \beta}-4 K^{\mu \nu} R_{\nu}^{\rho} \nabla_{\mu} \nabla_{\rho} \sigma+4 K^{\mu \nu} R_{\mu \alpha \beta \nu} \nabla^{\alpha} \nabla^{\beta} \sigma+\cdots\right], \\
\left(\sqrt{-g} K^{\mu \nu} R^{\alpha \beta} R_{\alpha \mu \beta \nu}\right)^{\prime}=\sqrt{-g}\left[K^{\alpha \beta} R^{\mu \nu} R_{\alpha \mu \beta \nu}+2 K^{\mu \nu} R_{\nu}^{\rho} \nabla_{\mu} \nabla_{\rho} \sigma-K^{\mu \nu} R \nabla_{\mu} \nabla_{\nu} \sigma\right. \\
\left.-K R^{\mu \nu} \nabla_{\mu} \nabla_{\nu} \sigma-K^{\mu \nu} R_{\mu \nu} \square \sigma+2 K^{\mu \nu} R_{\mu \alpha \beta \nu} \nabla^{\alpha} \nabla^{\beta} \sigma+\cdots\right], \\
\left(\sqrt{-g} K^{\mu \nu} R_{\mu \alpha} R_{\nu}^{\alpha}\right)^{\prime}=\sqrt{-g}\left[K^{\mu \nu} R_{\mu \alpha} R_{\nu}^{\alpha}-4 K^{\mu \nu} R_{\nu}^{\rho} \nabla_{\mu} \nabla_{\rho} \sigma-2 K^{\mu \nu} R_{\mu \nu} \square+\cdots\right], \\
\left(\sqrt{-g} R \nabla_{\mu} \nabla_{\nu} K^{\mu \nu}\right)^{\prime}=\sqrt{-g}\left[R \nabla_{\mu} \nabla_{\nu} K^{\mu \nu}-2 K^{\mu \nu} R \nabla_{\mu} \nabla_{\nu} \sigma-6 \nabla_{\mu} \nabla_{\nu} K^{\mu \nu} \square \sigma-6 K^{\mu \nu} \nabla_{\mu} R \nabla_{\nu} \sigma+K \nabla_{\rho} R \nabla^{\rho} \sigma+\cdots\right], \\
\left(\sqrt{-g} R_{\mu \nu} \square K^{\mu \nu}\right)^{\prime}=\sqrt{-g}\left[R_{\mu \nu} \square K^{\mu \nu}-4 K^{\mu \nu} R_{\nu}^{\rho} \nabla_{\mu} \nabla_{\rho} \sigma-2 K^{\mu \nu} R_{\mu \nu} \square \sigma-4 K^{\mu \nu} R_{\mu \alpha \beta \nu} \nabla^{\alpha} \nabla^{\beta} \sigma\right. \\
\left.-2 \nabla_{\mu} \nabla_{\nu} K^{\mu \nu} \square \sigma-2 K^{\mu \nu} \nabla_{\mu} R \nabla_{\nu} \sigma-K \square{ }^{2} \sigma+\cdots\right]
\end{gathered}
$$

and

$$
\begin{aligned}
{\left[-\sqrt{-g} \frac{K}{2}\left(\frac{1}{180} R_{\mu \nu \alpha \beta}^{2}-\frac{1}{180} R_{\mu \nu}^{2}+\frac{1}{180} \square R\right)\right]^{\prime}=} & \sqrt{-g}\left[-\frac{K}{2}\left(\frac{1}{180} R_{\mu \nu \alpha \beta}^{2}-\frac{1}{180} R_{\mu \nu}^{2}+\frac{1}{180} \square R\right)\right. \\
& \left.+\frac{1}{90} K R^{\mu \nu} \nabla_{\mu} \nabla_{\nu} \sigma-\frac{1}{180} K \nabla_{\rho} R \nabla^{\rho} \sigma+\frac{1}{60} K \square^{2} \sigma+\cdots\right] .
\end{aligned}
$$

Substituting the above formulas into (48), we find the conformal invariance (52).

[1] V. A. Kostelecký and S. Samuel, Spontaneous breaking of Lorentz symmetry in string theory, Phys. Rev. D 39, 683 (1989); Gravitational phenomenology in higher dimensional theories and strings, Phys. Rev. D 40, 1886 (1989); Phenomenological Gravitational Constraints on Strings and Higher Dimensional Theories, Phys. Rev. Lett. 63, 224 (1989); V. A. Kostelecký and R. Potting, CPT and strings, Nucl. Phys. B359, 545 (1991); V. A. Kostelecký and S. Samuel, Photon and Graviton Masses in String Theories, Phys. Rev. Lett. 66, 1811 (1991); V. A. Kostelecký and R. Potting, $C P T$, strings, and meson factories, Phys. Rev. D 51, 3923 (1995); Expectation values, Lorentz invariance, and $C P T$ in the open bosonic string, Phys. Lett. B 381, 89 (1996); V. A. Kostelecký, M. Perry, and R. Potting, OffShell Structure of the String Sigma Model, Phys. Rev. Lett. 84, 4541 (2000); V. A. Kostelecký and R. Potting, Analytical construction of a nonperturbative vacuum for the open bosonic string, Phys. Rev. D 63, 046007 (2001).

[2] R. Gambini and J. Pullin, Nonstandard optics from quantum space-time, Phys. Rev. D 59, 124021 (1999); J. Alfaro,
H. A. Morales-Tecotl, and L. F. Urrutia, Loop quantum gravity and light propagation, Phys. Rev. D 65, 103509 (2002).

[3] I. Mocioiu, M. Pospelov, and R. Roiban, Low-energy limits on the antisymmetric tensor field background on the brane and on the noncommutative scale, Phys. Lett. B 489, 390 (2000); S. M. Carroll, J. A. Harvey, V. A. Kostelecký, C. D. Lane, and T. Okamoto, Noncommutative Field Theory and Lorentz Violation, Phys. Rev. Lett. 87, 141601 (2001); Z. Guralnik, R. Jackiw, S. Y. Pi, and A.P. Polychronakos, Testing noncommutative QED, constructing noncommutative MHD, Phys. Lett. B 517, 450 (2001); A. Anisimov, T. Banks, M. Dine, and M. Graesser, Comments on noncommutative phenomenology, Phys. Rev. D 65, 085032 (2002); S. Aghababaei, M. Haghighat, and A. Kheirandish, Lorentz violation in the Higgs sector and the noncommutative standard model, Phys. Rev. D 87, 047703 (2013).

[4] I. L. Shapiro, Physical aspects of the space-time torsion, Phys. Rep. 357, 113 (2002); V. A. Kostelecký, N. Russell, and J. Tasson, New Constraints on Torsion from Lorentz Violation, Phys. Rev. Lett. 100, 111102 (2008). 
[5] J. Foster, V. A. Kostelecký, and R. Xu, Constraints on nonmetricity from bounds on Lorentz violation, Phys. Rev. D 95, 084033 (2017).

[6] B. Altschul and V. A. Kostelecký, Spontaneous Lorentz violation and nonpolynomial interactions, Phys. Lett. B 628, 106 (2005); M. Gomes, J. R. Nascimento, A. Y. Petrov, and A. J. da Silva, On the aether-like Lorentz-breaking actions, Phys. Rev. D 81, 045018 (2010).

[7] V. A. Kostelecký, Recent Progress in Lorentz and CPT Violation, arXiv:1610.09284.

[8] D. Colladay and V. A. Kostelecký, CPT violation and the standard model, Phys. Rev. D 55, 6760 (1997); Lorentz violating extension of the standard model, Phys. Rev. D 58, 116002 (1998); V. A. Kostelecký and R. Lehnert, Stability, causality, and Lorentz and CPT violation, Phys. Rev. D 63, 065008 (2001); D. Colladay and V. A. Kostelecký, Crosssections and Lorentz violation, Phys. Lett. B 511, 209 (2001).

[9] V. A. Kostelecký and N. Russell, Data tables for Lorentz and CPT violation, Rev. Mod. Phys. 83, 11 (2011).

[10] V. A. Kostelecký, C. D. Lane, and A. G. M. Pickering, One loop renormalization of Lorentz violating electrodynamics, Phys. Rev. D 65, 056006 (2002).

[11] V.A. Kostelecký, Gravity, Lorentz violation, and the standard model, Phys. Rev. D 69, 105009 (2004); V. A. Kostelecký and J. D. Tasson, Matter-gravity couplings and Lorentz violation, Phys. Rev. D 83, 016013 (2011).

[12] S. M. Christensen, Second- and fourth-order invariants on curved manifolds with torsion, J. Phys. A 13, 3001 (1980).

[13] I. L. Buchbinder, S. D. Odintsov, and I. L. Shapiro, Effective Action in Quantum Gravity (IOP Publishing, Bristol, 1992).

[14] G. de Berredo-Peixoto and I. L. Shapiro, On the renormalization of $C P T /$ Lorentz violating QED in curved space, Phys. Lett. B 642, 153 (2006).

[15] I. L. Shapiro, Renormalization in QED and QFT with a Lorentz- and $C P T$-violating backgrounds, Contribution to the proceedings of Sixth Meeting on CPT and Lorentz Symmetry, CPT'13, Indiana University (2013), arXiv:1309.4190.

[16] T. de P. Netto and I. L. Shapiro, Vacuum contribution of photons in the theory with Lorentz and CPT-violating terms, Phys. Rev. D 89, 104037 (2014).

[17] T. Appelquist and J. Carazzone, Infrared singularities and massive fields, Phys. Rev. D 11, 2856 (1975).

[18] E. V. Gorbar and I. L. Shapiro, Renormalization group and decoupling in curved space, J. High Energy Phys. 02 (2003) 021; Renormalization group and decoupling in curved space. 2. The standard model and beyond, J. High Energy Phys. 06 (2003) 004.

[19] N. D. Birrell and P.C.W. Davies, Quantum Fields in Curved Space (Cambridge University Press, Cambridge, 1982).

[20] F. L. Bezrukov and M. Shaposhnikov, The standard model Higgs boson as the inflaton, Phys. Lett. B 659, 703 (2008); F. L. Bezrukov, A. Magnin, and M. Shaposhnikov, Standard model Higgs boson mass from inflation, Phys. Lett. B 675, 88 (2009); F. Bezrukov and M. Shaposhnikov, Standard model Higgs boson mass from inflation: Two loop analysis, J. High Energy Phys. 07 (2009) 089.

[21] A. O. Barvinsky, A. Y. Kamenshchik, and A. A. Starobinsky, Inflation scenario via the standard model Higgs boson and
LHC, J. Cosmol. Astropart. Phys. 11 (2008) 021; A. O. Barvinsky, A. Y. Kamenshchik, C. Kiefer, A. A. Starobinsky, and C. Steinwachs, Asymptotic freedom in inflationary cosmology with a nonminimally coupled Higgs field, J. Cosmol. Astropart. Phys. 12 (2009) 003; , Higgs boson, renormalization group, and naturalness in cosmology, Eur. Phys. J. C 72, 2219 (2012).

[22] M. J. Duff, Twenty years of the Weyl anomaly, Classical Quantum Gravity 11, 1387 (1994).

[23] I. L. Shapiro, Local conformal symmetry and its fate at quantum level, Proc. Soc., IC2006 (2006) 030, arXiv: hep-th/0610168.

[24] R. J. Riegert, A nonlocal action for the trace anomaly, Phys. Lett. B 134, 56 (1984); E. S. Fradkin and A. A. Tseytlin, Conformal anomaly in Weyl theory and anomaly free superconformal theories, Phys. Lett. B 134, 187 (1984).

[25] A. A. Starobinsky, A new type of isotropic cosmological models without singularity, Phys. Lett. B 91, 99 (1980); Nonsingular model of the universe with the quantum gravitational de Sitter stage and its observational consequences, Proceedings of the second seminar, Quantum theory of gravity (INR Press, Moscow, 1982), p. 58.

[26] J. C. Fabris, A. M. Pelinson, and I. L. Shapiro, Anomaly induced effective action for gravity and inflation, Gravitation Cosmol. 6, 59 (2000); I. L. Shapiro and J. Sola, Massive fields temper anomaly induced inflation, Phys. Lett. B 530, 10 (2002); I. L. Shapiro, The graceful exit from the anomaly induced inflation: Supersymmetry as a key, Int. J. Mod. Phys. D 11, 1159 (2002); A. M. Pelinson, I. L. Shapiro, and F. I. Takakura, On the stability of the anomaly induced inflation, Nucl. Phys. B648, 417 (2003); Stability issues in the modified Starobinsky model, Nucl. Phys. (PS)127, 182 (2004); T. de P. Netto, A. M. Pelinson, I. L. Shapiro, and A. A. Starobinsky, From stable to unstable anomaly-induced inflation, Eur. Phys. J. C 76, 544 (2016).

[27] V. A. Kostelecký and M. Mewes, Lorentz-Violating Electrodynamics and the Cosmic Microwave Background, Phys. Rev. Lett. 99, 011601 (2007).

[28] B.S. DeWitt, Dynamical Theory of Groups and Fields (Gordon and Breach, New York, 1965).

[29] A. O. Barvinsky and G. A. Vilkovisky, The generalized Schwinger-DeWitt technique in gauge theories and quantum gravity, Phys. Rep. 119, 1 (1985).

[30] B. Altschul, Lorentz violation and the Yukawa potential, Phys. Lett. B 639, 679 (2006); Lorentz and CPT violation in scalar-mediated potentials, Phys. Rev. D 87, 045012 (2013).

[31] D. L. Anderson, M. Sher, and I. Turan, Lorentz and CPT violation in the Higgs sector, Phys. Rev. D 70, 016001 (2004).

[32] A. Ferrero and B. Altschul, Renormalization of scalar and Yukawa field theories with Lorentz violation, Phys. Rev. D 84, 065030 (2011); A. Ferrero, Solution to the $\beta$-functions in Lorentz-violating theories as a decomposition into irreducible representations, Phys. Rev. D 91, 125012 (2015).

[33] B. Altschul, Lorentz violation and the Higgs mechanism, Phys. Rev. D 86, 045008 (2012).

[34] P. R. S. Carvalho, Mass renormalization in Lorentzviolating scalar field theory, Phys. Lett. B 726, 850 (2013); Three-loop field renormalization for scalar field theory with Lorentz violation, Phys. Lett. B 730, 320 (2014). 
[35] W. de Carvalho Vieira and P. R. Silva de Carvalho, Renormalization group approach for Lorentz-violating scalar field theory at all loop orders, Int. J. Geom. Methods Mod. Phys. 13, 1650049 (2016).

[36] J. R. Nascimento, A. Y. Petrov, and C. M. Reyes, Renormalization in a Lorentz violating model and higher-order operators, arXiv:1706.01466.

[37] A. P. Baeta Scarpelli, L. C. T. Brito, J. C. C. Felipe, J. R. Nascimento, and A. Y. Petrov, Effective potential in Lorentz breaking field theory models, Eur. Phys. J. C 77, 850 (2017).

[38] M. Frank and I. Turan, The Casimir force in a Lorentz violating theory, Phys. Rev. D 74, 033016 (2006); M. B. Cruz, E. R. Bezerra de Mello, and A. Y. Petrov, Casimir effects in Lorentz-violating scalar field theory, Phys. Rev. D 96, 045019 (2017).

[39] D. Bazeia and R. Menezes, Defect structures in Lorentz and CPT violating scenarios, Phys. Rev. D 73, 065015 (2006).

[40] R. Casana and K. A. T. da Silva, Lorentz-violating effects in the Bose-Einstein condensation of an ideal bosonic gas, Mod. Phys. Lett. A 30, 1550037 (2015).

[41] I. L. Buchbinder, T. de P. Netto, and I. L. Shapiro, Massive vector field on curved background: Nonminimal coupling, quantization, and divergences, Phys. Rev. D 95, 085009 (2017).

[42] Q. G. Bailey and V. A. Kostelecký, Signals for Lorentz violation in post-Newtonian gravity, Phys. Rev. D 74, 045001 (2006).

[43] Q. G. Bailey, V. A. Kostelecký, and R. Xu, Short-range gravity and Lorentz violation, Phys. Rev. D 91, 022006 (2015); J. C. Long and V. A. Kostelecký, Search for Lorentz violation in short-range gravity, Phys. Rev. D 91, 092003 (2015); C. G. Shao et al., Combined Search for Lorentz Violation in Short-Range Gravity, Phys. Rev. Lett. 117, 071102 (2016); Q. G. Bailey, Anisotropic cubic curvature couplings, Phys. Rev. D 94, 065029 (2016); A. Hees, Q. G. Bailey, A. Bourgoin, H. P. L. Bars, C. Guerlin, and C. Le Poncin-Lafitte, Tests of Lorentz symmetry in the gravitational sector, Universe 2, 30 (2016); V. A. Kostelecký and M. Mewes, Testing local Lorentz invariance with shortrange gravity, Phys. Lett. B 766, 137 (2017).

[44] C. Hernaski and H. Belich, Lorentz violation and higherderivative gravity, Phys. Rev. D 89, 104027 (2014).

[45] M. J. Duff, Observations on conformal anomalies, Nucl. Phys. B125, 334 (1977).

[46] I. L. Shapiro, Effective action of vacuum: Semiclassical approach, Classical Quantum Gravity 25, 103001 (2008).

[47] S. M. Christensen, Vacuum expectation value of the stress tensor in an arbitrary curved background: The covariant point separation method, Phys. Rev. D 14, 2490 (1976); Regularization, renormalization, and covariant geodesic point separation, Phys. Rev. D 17, 946 (1978).

[48] S. Deser, M. J. Duff, and C. J. Isham, Nonlocal conformal anomalies, Nucl. Phys. B111, 45 (1976).
[49] M. Asorey, E. V. Gorbar, and I. L. Shapiro, Universality and ambiguities of the conformal anomaly, Classical Quantum Gravity 21, 163 (2004); M. Asorey, G. de BerredoPeixoto, and I. L. Shapiro, Renormalization ambiguities and conformal anomaly in metric-scalar backgrounds, Phys. Rev. D 74, 124011 (2006).

[50] A. G. Mirzabekian, G. A. Vilkovisky, and V. V. Zhytnikov, Partial summation of the nonlocal expansion for the gravitational effective action in four-dimensions, Phys. Lett. B 369, 215 (1996).

[51] D. F. Carneiro, E. A. Freiras, B. Gonçalves, A. G. de Lima, and I. L. Shapiro, On useful conformal transformations in general relativity, Gravitation Cosmol. 10, 305 (2004).

[52] S. Paneitz, A quartic conformally covariant differential operator for arbitrary pseudo-Riemannian manifolds, SIGMA 4, 036 (2008).

[53] A. A. Starobinsky, Relict gravitation radiation spectrum and initial state of the universe, JETP Lett. 30, 682 (1979); The perturbation spectrum evolving from a nonsingular initially de-Sitter cosmology and the microwave background Anisotropy, Sov. Astron. Lett. 9, 302 (1983).

[54] J. C. Fabris, A. M. Pelinson, and I. L. Shapiro, On the gravitational waves on the background of anomaly-induced inflation, Nucl. Phys. B597, 539 (2001); J. C. Fabris, A. M. Pelinson, F. de O. Salles, and I. L. Shapiro, Gravitational waves and stability of cosmological solutions in the theory with anomaly-induced corrections, J. Cosmol. Astropart. Phys. 02 (2012) 019; F. de O. Salles and I. L. Shapiro, Do we have unitary and (super)renormalizable quantum gravity below the Planck scale?, Phys. Rev. D 89, 084054 (2014); I. L. Shapiro, A. M. Pelinson, and F. de O. Salles, Gravitational waves and perspectives for quantum gravity, Mod. Phys. Lett. A 29, 1430034 (2014); G. Cusin, F. de O. Salles, and I. L. Shapiro, Tensor instabilities at the end of the $\Lambda$ CDM universe, Phys. Rev. D 93, 044039 (2016).

[55] S. W. Hawking, T. Hertog, and H. S. Reall, Trace anomaly driven inflation, Phys. Rev. D 63, 083504 (2001).

[56] R. Balbinot, A. Fabbri, and I. L. Shapiro, Anomaly Induced Effective Actions and Hawking Radiation, Phys. Rev. Lett. 83, 1494 (1999); Vacuum polarization in Schwarzschild space-time by anomaly induced effective actions, Nucl. Phys. B559, 301 (1999); P. R. Anderson, E. Mottola, and R. Vaulin, Stress tensor from the trace anomaly in Reissner-Nordstrom spacetimes, Phys. Rev. D 76, 124028 (2007).

[57] I. L. Shapiro and A. G. Zheksenaev, Gauge dependence in higher derivative quantum gravity and the conformal anomaly problem, Phys. Lett. B 324, 286 (1994); P. O. Mazur and E. Mottola, Weyl cohomology and the effective action for conformal anomalies, Phys. Rev. D 64, 104022 (2001).

[58] S. M. Christensen and S. A. Fulling, Trace anomalies and the Hawking effect, Phys. Rev. D 15, 2088 (1977). 